\title{
Enhanced Living Environments
}

From models to technologies

\section{Edited by}

Rossitza Ivanova Goleva, Ivan Ganchev, Ciprian Dobre, Nuno Garcia and Carlos Valderrama

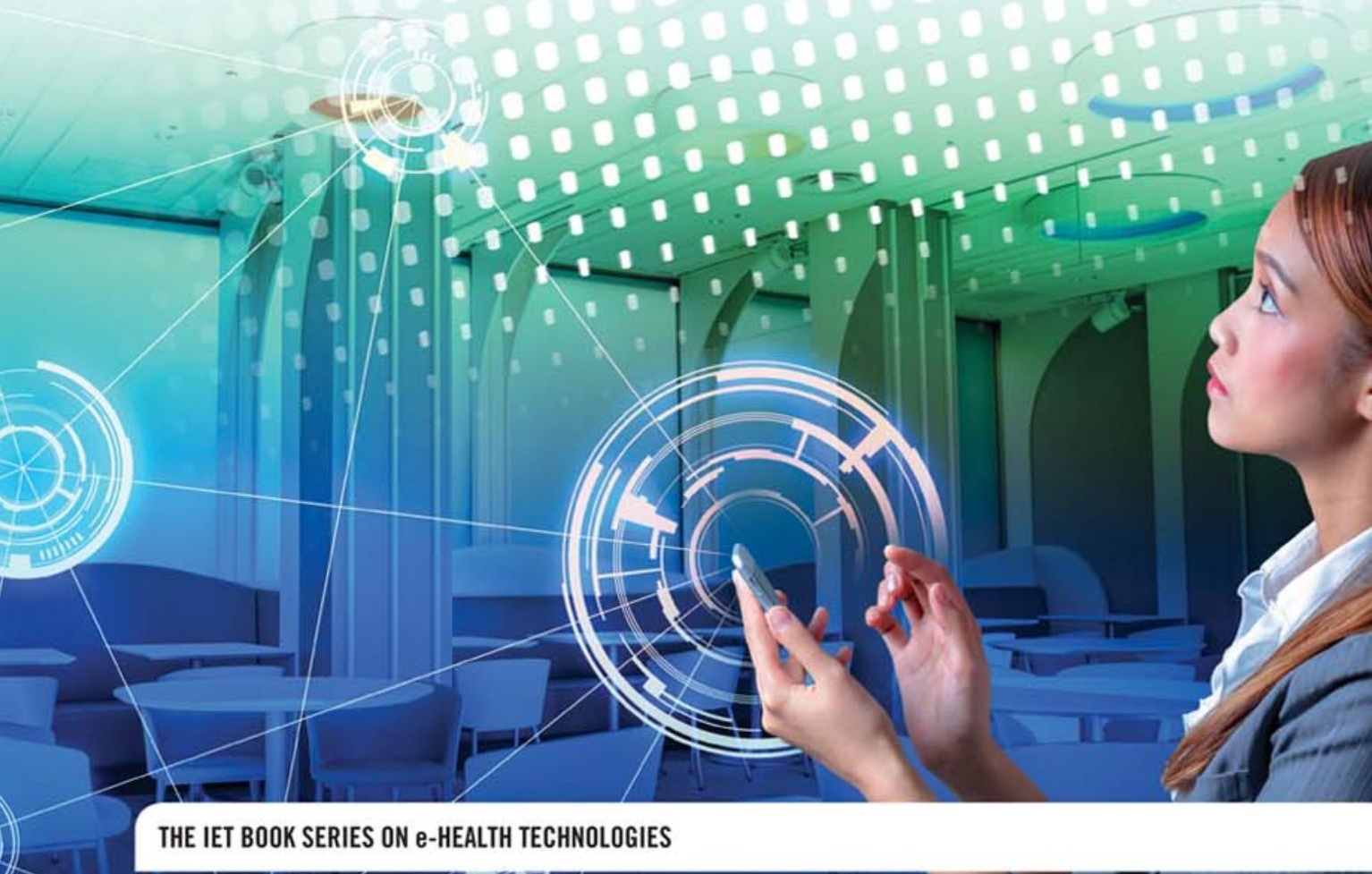


IET HEALTHCARE TECHNOLOGIES SERIES 10

\section{Enhanced Living \\ Environments}




\section{IET book series on e-health technologies - call for authors}

Book Series Editor: Professor Joel P. C. Rodrigues, the National Institute of Telecommunications (Inatel), Brazil and Instituto de Telecomunicações, Portugal

While the demographic shifts in populations display significant socioeconomic challenges, they trigger opportunities for innovations in e-Health, $\mathrm{m}$-Health, precision and personalized medicine, robotics, sensing, the Internet of Things, cloud computing, Big Data, software-defined networks and network function virtualization. Their integration is however associated with many technological, ethical, legal, social and security issues. This new Book Series aims to disseminate recent advances for e-Health Technologies to improve healthcare and people's well-being.

Topics considered include Intelligent e-Health systems, electronic health records, ICT-enabled personal health systems, mobile and cloud computing for eHealth, health monitoring, precision and personalized health, robotics for e-Health, security and privacy in e-Health, ambient assisted living, telemedicine, Big Data and IoT for e-Health, and more.

Proposals for coherently integrated international multi-authored edited or co-authored handbooks and research monographs will be considered for this Book Series. Each proposal will be reviewed by the Book Series Editor with additional external reviews from independent reviewers. Please email your book proposal for the IET Book Series on e-Health Technologies to: Professor Joel Rodrigues at joeljr@ieee.org or joeljr@inatel.br 


\section{Enhanced Living Environments}

From models to technologies

Edited by

Rossitza Ivanova Goleva, Ivan Ganchev, Ciprian Dobre, Nuno Garcia and Carlos Valderrama 
Published by The Institution of Engineering and Technology, London, United Kingdom The Institution of Engineering and Technology is registered as a Charity in England \& Wales (no. 211014) and Scotland (no. SC038698).

(c) The Institution of Engineering and Technology 2018

First published 2017

This publication is copyright under the Berne Convention and the Universal Copyright Convention. All rights reserved. Apart from any fair dealing for the purposes of research or private study, or criticism or review, as permitted under the Copyright, Designs and Patents Act 1988, this publication may be reproduced, stored or transmitted, in any form or by any means, only with the prior permission in writing of the publishers, or in the case of reprographic reproduction in accordance with the terms of licences issued by the Copyright Licensing Agency. Enquiries concerning reproduction outside those terms should be sent to the publisher at the undermentioned address:

The Institution of Engineering and Technology

Michael Faraday House

Six Hills Way, Stevenage

Herts, SG1 2AY, United Kingdom

www.theiet.org

While the authors and publisher believe that the information and guidance given in this work are correct, all parties must rely upon their own skill and judgement when making use of them. Neither the authors nor publisher assumes any liability to anyone for any loss or damage caused by any error or omission in the work, whether such an error or omission is the result of negligence or any other cause. Any and all such liability is disclaimed.

The moral rights of the authors to be identified as authors of this work have been asserted by them in accordance with the Copyright, Designs and Patents Act 1988.

\section{British Library Cataloguing in Publication Data}

A catalogue record for this product is available from the British Library

ISBN 978-1-78561-211-4 (hardback)

ISBN 978-1-78561-212-1 (PDF)

Typeset in India by MPS Limited

Printed in the UK by CPI Group (UK) Ltd, Croydon 


\section{Dedication}

To my family and friends.

In Memoriam to Rumen Stainov.

- Rossitza Goleva

To all my family and extended family.

- Ivan Ganchev

To Anamaria and Iulia, the two beautiful girls in my life, with all my love.

- Ciprian Dobre

To the true team spirit of the AAPELE Team

and to my family and friends!

- Nuno M. Garcia

To the great AAPELE team that provides the essential energy we all need as well as to our families who support our dedication and sacrifices.

- Carlos Valderrama 
This page intentionally left blank 


\section{Contents}

$\begin{array}{lr}\text { About the editors } & \text { xv } \\ \text { Preface } & \text { xix } \\ \text { Acknowledgements } & \text { xxiii }\end{array}$

1 Introduction to enhanced living environments 1

Ciprian Dobre, Ivan Ganchev, Nuno M. Garcia, Rossitza Goleva and Carlos Alberto Valderrama

Abstract

1.1 Introduction

1.2 An overview of healthcare systems

1.3 Ambient assisted living and enhanced living environments

1.4 Conclusions

Acknowledgements

References

Biographies

2 Enhanced living environments from the viewpoint of socioecological psychology

Tamás Martos and Viola Sallay

Abstract

2.1 Introduction

2.2 Socioecological psychology as a framework

2.3 Concept of 'human niche construction' as a universal human phenomenon

2.4 Home as a niche

2.4.1 Emotional processes in the home: lessons learnt with the Emotional Map of the Home Interview

2.5 Legacy of Self-determination theory

2.6 Putting it all together: socioecological psychological aspects of ELE

2.6.1 ELE as niche construction

2.6.2 ELE and home niches: the potential places of technology in a complex system

2.6.3 Constructing ELE solutions in home niches: the importance of psychological need support 
2.7 Conclusions

Acknowledgements $\quad 41$

References 41

Further reading $\quad 46$

$\begin{array}{ll}\text { Biographies } & 47\end{array}$

3 Pervasive sensing for social connectedness $\quad 49$

Kadian Davis, Evans B. Owusu, Lucio Marcenaro, Jun Hu, Carlo S. Regazzoni, and Loe Feijs

Abstract 49

3.1 Introduction 49

3.1.1 Social isolation and loneliness as risk factors $\quad 50$

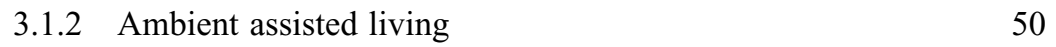

3.2 A user-centred approach for designing systems to support social connectedness $\quad 52$

3.2.1 A user-centred design process 53

3.3 Context-aware systems for social connectedness 55

3.4 Pervasive sensing and models for HAR 57

3.5 A case study evaluating the HMM-SVM model 60

$\begin{array}{lll}3.6 & \text { Context-aware connectedness systems } & 62\end{array}$

$\begin{array}{lll}3.7 & \text { Experimental results } & 65\end{array}$

3.7.1 Perceptions on context-aware solutions for social connectedness 65

3.7.2 HAR-based activity displays for social connectedness 66

$\begin{array}{lll}3.8 & \text { Challenges } & 69\end{array}$

3.9 Conclusion $\quad 69$

$\begin{array}{ll}\text { Acknowledgements } & 70\end{array}$

$\begin{array}{ll}\text { References } & 70\end{array}$

$\begin{array}{ll}\text { Further reading } & 77\end{array}$

$\begin{array}{ll}\text { Data set } & 77\end{array}$

$\begin{array}{ll}\text { Biographies } & 77\end{array}$

4 Ethics in information and communication technologies: training the elderly in making gerontechnology accessible $\quad 81$

Hélène Geurts, Marie-Claire Haelewyck, and Carlos Valderrama

$\begin{array}{ll}\text { Abstract } & 81\end{array}$

4.1 Introduction $\quad 81$

$\begin{array}{lll}4.2 & \text { Generation effect } & 82\end{array}$

4.3 Gerontechnology is a neologism vector of the future 83

$\begin{array}{lll}4.4 & \text { Pitfalls to be avoided } & 85\end{array}$

$\begin{array}{lll}4.5 & \text { Ethical issues at stake } & 88\end{array}$

4.6 Identification of needs, the keystone of reflection 90

4.7 Need for acceptability, the secret of success 93

$\begin{array}{lll}4.8 \text { Conclusion } & 95\end{array}$

Acknowledgements $\quad 95$ 
$\begin{array}{lr}\text { References } & 95\end{array}$

$\begin{array}{ll}\text { Further reading } & 98\end{array}$

Biographies $\quad 99$

5 End-users' AAL and ELE service scenarios in smart personal environments

Serge Autexier, Rossitza Goleva, Nuno M. Garcia, Rumen Stainov, Ivan Ganchev', Constandinos X. Mavromoustakis, Ciprian Dobre, Ivan Chorbev, Vladimir Trajkovik, and Eftim Zdravevski

Abstract

5.1 Introduction

5.2 State of the art

5.3 Living lab architecture

5.4 End-user groups

5.5 From single user and single sensor to the cloud and back

5.6 Scenarios

5.7 Customized ELE ICT services

5.8 Conclusions and further research directions

Acknowledgements

References

Further reading

Biographies

6 Technological support to stress-level monitoring

\section{Valentina Markova and Todor Ganchev}

Abstract

6.1 Introduction

6.2 State-of-the-art personal health monitoring systems

6.2.1 Physiological parameters and stress

6.2.2 Overview of system architectures

6.2.3 Short-range wireless network technology

6.3 Stress and emotion assessment

6.3.1 Stress assessment procedure 146

$\begin{array}{ll}\text { 6.3.2 Emotion recognition } & 147\end{array}$

$\begin{array}{lll}6.4 & \text { Use cases } & 148\end{array}$

$\begin{array}{lll}6.4 .1 & \text { Stationary setup } & 148\end{array}$

$\begin{array}{ll}\text { 6.4.2 Mobile setup } & 149\end{array}$

$\begin{array}{lll}6.4 .3 & \text { Recent projects } & 151\end{array}$

6.4.4 SLADE application scenario 152

6.5 Future technology in support of stress monitoring and management

References

Further reading 
7 Big data healthcare system to improve healthcare information searching in the Internet

Mariya Savova Evtimova

Abstract

7.1 Introduction

$\begin{array}{lll}7.2 & \text { Intelligent agents' advantages and characteristics } & 162\end{array}$

7.3 Fuzzy logic and probability

7.4 Meaning of big data in a personalized search of uncertain and vague information

$\begin{array}{lll}\text { 7.4.1 Value and demand in-depth analysis } & 165\end{array}$

$\begin{array}{ll}\text { 7.4.2 Variety and heterogeneity of data } & 165\end{array}$

$\begin{array}{ll}\text { 7.4.3 Quality of data } & 166\end{array}$

7.4.4 Volume and size of data 166

7.4.5 Speed and timeliness of the data 166

$\begin{array}{lll}7.5 & \text { Rule-based and case-based reasoning } & 166\end{array}$

$\begin{array}{lll}7.6 & \text { Related work } & 167\end{array}$

$\begin{array}{lll}\text { 7.7 Agent-based system for personalized searching } & 167\end{array}$

7.7.1 Aims and tasks of the developed system 168

7.7.2 Conceptual model for personalized semantic search system when the information in the query is fuzzy and uncertain

7.8 Concept of building a customized profile information

7.9 Development of applied subjective ontology: problems and approaches

7.9.1 Storing the knowledge in the fuzzy ontology of the proposed semantic system

7.9.2 Fuzzification process in case-based ontology 174

7.9.3 Design of the fuzzy and vague case-based ontology 174

7.10 Description of the process of reasoning 175

7.11 Metrics for evaluating the quality of the returned results $\begin{array}{ll}\text { from the search system } & 178\end{array}$

$\begin{array}{ll}7.12 \text { Conclusions } & 178\end{array}$

References 180

Further reading 182

List of abbreviations 182

Biography 182

8 Sensors for wireless body area networks 183

Ivelina Nikolaeva Ruskova and Elitsa Emilova Gieva 
8.1 Introduction: wireless body area networks and wireless sensor network

8.2 Sensor node

8.3 Overview of sensor characteristics

8.4 WBAN technologies

8.4.1 Applications depending on the technology

8.5 Conclusion

References

Further reading

Biographies

9 AALaaS/ELEaaS platforms

Rossitza Goleva, Mara Pudane, Sintija Petrovica, Egons Lavendelis, Karl Kreiner, Mario Drobics, Ivan Ganchev, Nuno M. Garcia, Rumen Stainov, Ciprian Dobre, Constandinos X. Mavromoustakis, Ivan Chorbev, Vladimir Trajkovik, Eftim Zdravevski, and George Mastorakis
Abstract
9.1 Introduction
9.2 State of the art
9.3 Generic AALaaS/ELEaaS architecture
9.4 Affective computing mapping implementation
9.5 KIOLA platform implementation
9.6 AAL/ELE laboratory and home implementation
9.7 Conclusion and further research plan
Acknowledgements
References
Further reading
Biographies

10 Linear wireless sensor networks and protocols in the next-generation networks

Radosveta I. Sokullu and Eren Demir

Abstract

10.1 Introduction

10.2 Linear wireless sensor networks

10.2.1 Network model

10.2.2 Variations of LWSNs

10.2.3 Objectives and challenges of LWSNs

10.3 MAC protocols for LWSNs

10.4 Open research issues

10.5 Conclusion 
Further reading

List of abbreviations $\quad 266$

\begin{tabular}{lr} 
Glossary & 267 \\
\hline & 266
\end{tabular}

$\begin{array}{ll}\text { Biographies } & 268\end{array}$

11 Model-compilation challenges for cyber-physical systems

Belgacem Ben Hedia, Chokri Mraidha, Etienne Hamelin, and Sara Tucci-Piergiovanni

$\begin{array}{ll}\text { Abstract } & 269\end{array}$

$\begin{array}{lll}11.1 & \text { Introduction } & 269\end{array}$

$\begin{array}{lll}11.2 & \text { CPS challenges } & 272\end{array}$

$\begin{array}{lll}11.3 & \text { Model-compilation methodology and approach } & 275\end{array}$

11.3.1 Front-end: from multiple heterogeneous high-level models $\quad 275$

11.3.2 Middle-end: model-compilation into SwArch 280

11.3.3 Back-end: transformation into concrete target platforms 283

11.3.4 Design iterations 283

11.4 Model-compilation methodology assessment 284

11.4.1 Applicability of model-compilation approach 284

$\begin{array}{ll}\text { 11.4.2 Productivity enhancements } & 285\end{array}$

$\begin{array}{lll}11.5 & \text { Related works } & 285\end{array}$

11.5.1 Model-based methodologies for safety
and timing

11.5.2 Model-compilation $\quad 286$

$\begin{array}{lll}\text { 11.5.3 Physical modelling } & 287\end{array}$

$\begin{array}{lll}11.6 & \text { Conclusion } & 288\end{array}$

$\begin{array}{ll}\text { References } & 288\end{array}$

$\begin{array}{ll}\text { Biographies } & 291\end{array}$

12 Health monitoring using WBAN: topology design, routing and thermal issues

Ghufran Ahmed, Saif Ul Islam, Maham Shahid, Azfar Shakeel, Najmun Nisa, Najmul Hassan, Numera M.I. Shahid,

Zaheer Ul Hussain Sani, and Hilal Jan

Abstract

12.1 Introduction 293

12.1.1 Applications $\quad 295$

$\begin{array}{ll}\text { 12.1.2 WBAN wireless technologies } & 298\end{array}$

12.1.3 WBAN infrastructure $\quad 300$

$\begin{array}{lll}12.1 .4 & \text { Energy efficiency } & 300\end{array}$

12.1.5 Approaches to achieve energy efficiency 300

$\begin{array}{lll}12.2 & \text { Energy-aware topology design } & 301\end{array}$

12.2.1 Optimization of relay nodes placement 304 
12.3 SAR analysis

12.3.1 Using low transmission power level to reduce SAR

12.3.2 Impact of frequency band on SAR values 305

12.3.3 Impact of high SAR on human body 305

$\begin{array}{lll}12.4 & \text { Energy efficient and SAR-aware routing } 306\end{array}$

12.4.1 Energy-efficient routing 306

$\begin{array}{lll}\text { 12.4.2 SAR-aware routing } & 308\end{array}$

$\begin{array}{ll}12.5 \text { Conclusion } & 309\end{array}$

References $\quad 309$

$\begin{array}{ll}\text { Biographies } & 313\end{array}$

13 Wearable health care: technology evolution, algorithms and needs

Raluca Maria Aileni, Sever Pasca, Carlos Alberto Valderrama, and Rodica Strungaru

Abstract

13.1 Introduction

13.2 Wearable technology evolution

13.3 Healthcare perspectives for wearable devices

13.4 Algorithms dedicated to wearable technologies

13.4.1 Case 1: wearable sensors for body temperature monitoring

13.4.2 Case 2: wearable sensors for human skin conductance response

13.4.3 Case 3: wearable sensors for human activity monitoring

13.5 Wearable: user needs and expectations 334

$\begin{array}{lll}13.6 & \text { Future wearable challenges } & 335\end{array}$

$\begin{array}{lll}13.7 & \text { Conclusions } & 337\end{array}$

$\begin{array}{ll}\text { Acknowledgements } & 338\end{array}$

$\begin{array}{lr}\text { References } & 338\end{array}$

Biographies $\quad 342$

14 Intelligent system for after-stroke home rehabilitation $\mathbf{3 4 5}$

Nirvana Popescu, Marian-Silviu Poboroniuc, Decebal Popescu,

Dănuţ Irimia, and Alexandru Valer Grigoraş

Abstract

$\begin{array}{lll}14.1 & \text { Introduction } & 345\end{array}$

14.2 Design and development of the IHRG structure 348

$\begin{array}{lll}14.3 & \text { Voice control approach } & 349\end{array}$

14.3.1 Hardware and software design 349

14.3.2 Experiments with vocal commands 352

14.4 Predefined recovery exercises system for home use 353 
xiv Enhanced living environments: from models to technologies

14.5 Hybrid FES-robotic glove approach 354

14.5.1 Hybrid system description $\quad 354$

14.5.2 Experimental results 358

$\begin{array}{lll}\text { 14.5.3 Statistical analysis } & 360\end{array}$

$\begin{array}{lll}14.6 & \text { Conclusion } & 364\end{array}$

References $\quad 365$

Biographies $\quad 366$

Index 


\section{About the editors}

Ciprian Dobre is Professor within the Computer Science Department, University Politehnica of Bucharest (Habil. since 2014, Dr. Since 2008 with Cum laudae). He currently leads the activities within Laboratory on Pervasive products and services, and MobyLab. Ciprian Dobre's research interests involve research subjects related to mobile wireless networks and computing applications, pervasive services, context awareness and people-centric or participatory sensing. He has scientific and scholarly contributions in the field of large-scale distributed systems concerning mobile applications and smart technologies to reduce urban congestion and air pollution (MobiWay, TRANSYS), context-aware applications (CAPIM), opportunistic networks and mobile data offloading (SPRINT, SENSE), monitoring (MonALISA), high-speed networking (VINCI, FDT), Grid application development (EGEE, SEE-GRID) and evaluation using modelling and simulation (MONARC 2, VNSim). These contributions led to important results, demonstrating his qualifications and potential to go significantly beyond the state of the art. Ciprian Dobre was awarded a PhD scholarship from California Institute of Technology (Caltech, USA), and another one from Oracle. His results received one IBM Faculty Award, two CENIC Awards and three Best Paper Awards (in 2013, 2012 and 2010). The results were published in over 100 chapters in edited books, articles in major international peer-reviewed journals and papers in well-established international conferences and workshops.

Dr. Ivan Ganchev is a Senior Member of the Institute of Electrical and Electronic Engineers (IEEE), the IEEE Communications Society, the IEEE Consumer Electronics Society, the IEEE Internet of Things Community, the IEEE Smart Cities Community and the IEEE Consultants Network. He received his doctoral and engineering (summa cum laude) degrees from the Saint-Petersburg State University of Telecommunications. He is a Deputy Director of the Telecommunications Research Centre (TRC), University of Limerick (Ireland), an Associate Professor from the University of Plovdiv "Paisii Hilendarski", an ITU-T Invited Expert and an IET Invited Lecturer. Dr. Ganchev was involved in 35+ international and national research and education projects. His research interests include novel telecommunications paradigms, future networks and services, smart ubiquitous networking, context-aware networking, mobile cloud computing, Internet of Things (IoT), Internet of Services (IoS), ambient assisted living (AAL), enhanced living environments (ELE), trust management, Internet tomography, mHealth and mLearning ICT. Dr Ganchev has served on the Technical Program Committee of 
$200+$ prestigious international conferences, symposia and workshops. He has authored/co-authored 6 books (including 2 edited books) and 240+ research papers in refereed international journals and conference proceedings. Dr. Ganchev is on the editorial board of and has served as a Guest Editor for multiple international journals.

Nuno M. Garcia holds a Ph.D. in Computer Science Engineering from the University of Beira Interior (UBI, Covilhã, Portugal) (2008) and he is a 5-year B.Sc. (Hons.) in Mathematics/Informatics also from UBI (1999-2004). He is Assistant Professor at UBI and Invited Associate Professor at the School of Communication, Architecture, Arts and Information Technologies of the Universidade Lusófona de Humanidades e Tecnologias (Lisbon, Portugal). He was founder and is coordinator of the Assisted Living Computing and Telecommunications Laboratory (ALLab), a research group within the Instituto de Telecomunicações at UBI. He was also cofounder and is Coordinator of the Executive Council of the BSAFE LAB - Law enforcement, Justice and Public Safety Research and Technology Transfer Laboratory, a multidisciplinary research laboratory in UBI. He is the coordinator of the Cisco Academy at UBI, Head of EyeSeeLab in Eye- See Lda. (Lisbon, Portugal), and member of the Consultative Council of Favvus IT HR SA (Lisbon). He is also Chair of the COST Action IC1303 AAPELE - Architectures, Algorithms and Platforms for Enhanced Living Environments. He is the main author of several international, European and Portuguese patents. He is member of the Non-Commercial Users Constituency, a group within GNSO in ICANN. His main interests include next-generation networks, algorithms for bio-signal processing, distributed and cooperative protocols.

Rossitza Goleva received her Ph.D. in Communication Networks in 2016 and M.Sc. in Computer Science in 1982 at Technical University of Sofia, Bulgaria. She was part of the research staff of the research Institute of Bulgarian PTT between 1982 and 1987. Since 1987, she is with Department of Communication Networks at Technical University of Sofia. At present, Rossitza works on communication networks, communication protocols and software engineering. Her research interests are in Quality of Service in communication networks, communication protocols, traffic engineering, cloud and fog computing, performance analyses. She is an IEEE Member, involved in IEEE Bulgaria section activities, has more than 85 research publications, was part of more than 30 research projects including and EU COST IC1303 AAPELE action.

Carlos Alberto Valderrama is, since 2004, Director of the Electronics and Microelectronics Department at the Polytechnic Faculty of the University of Mons in Belgium. The Electronics and Microelectronics Department is member of the Numediart (New Media Art Technology) and the InforTec (Information Technology) Institutes. He obtained the Ph.D. degree in Microelectronics at the INPG in Grenoble, France, in 1998, the M.Sc. diploma at the Federal University of Rio de Janeiro (UFRJ-COPPE), Brazil, in 1993, and the electrical-electronics engineering 
diploma at the National University of Cordoba (UNC), Argentina, in 1989. He was visiting professor at two Brazilian universities, at the Federal University of Pernambuco (UFPE) in 2004 and at the Federal University of Rio Grande do Norte (UFRN) in 1998. Between 1999 and 2004, he was leading the Hardware-Flow team in CoWare NV. (today acquired by Synopsys), Belgium. In 2009, he was responsible for the creation of the spinoff Nsilition (http:/www.nsilition.com/) resulting from the IPfundry project funded by the Walloon Region. His primary research interests include wireless communication, EDA and system level design of reconfigurable embedded architectures. He has participated in more than 15 national and international research projects from the development of $4 \mathrm{G}$ chips to next-generation tracking devices and software architectures for the IoT, satellite and multicore industry, collaborating with partners such as Alcatel, STMicroelectronics, NXP and Thales. He serves as technical reviewer and committee member of multiple journals and international conferences. His research activity is supported by more than 150 publications on international conferences, more than 10 books chapters, and more than 30 scientific journals. He is IEEE senior member since 2006. 
This page intentionally left blank 


\section{Preface}

The implementation of the information and communication technologies (ICTs) in healthcare and caregiving areas, the increase in medical expenses, problems with demographic ageing, the dynamics of the everyday life and the necessity to take care of the family put a strong pressure on the sustainability of health and social care systems, on the labour participation and on the quality of life for elderly, people with disabilities, children and active people. Enhanced living environments (ELEs) encompass all ICT technological achievements supporting true ambientassisted living (AAL) including capabilities for better living and healthcare organization. ELE promotes the provision of infrastructures and services for better living via the seamless integration of ICT within homes and residences, thus increasing the quality of life of human beings, maintaining one's home as a preferable living environment for as long as possible and not causing disruption in the web of social and family interactions. AAL aims to construct safe environments around assisted peoples (and local/remote caregivers/family members) in order to help them maintain independent and/or more autonomous lifestyle. Finally, AAL/ ELE technologies allow the creation of personal living environments (PLEs) concerned with not only medical or fitness related functionalities but also social isolation, stress level, emotional state, etc.

Most efforts today towards the realization of AAL/ELE systems are based on developing specialized devices and the use of ambient intelligence to integrate these devices together to construct a safe PLE. There is a missing interaction of multiple stakeholders needing to collaborate for ELE supporting a multitude of AAL services, as well as barriers to innovation in the markets concerned, the governments, and health care sector. These innovations do not yet take place at a relevant scale.

Many fundamental issues in the ELE area remain open. Most of the current efforts still do not fully express the power of human being, and the importance of social connections and societal activities is less noticed. Effective ELE solutions require appropriate ICT algorithms, architectures and platforms, having in view the advance of science in this area and the development of new and innovative connected solutions (particularly in the area of smart dust/dew/fog/cloud computing). In this sense, the book aims to provide a platform for the dissemination of research efforts and presentation of advances in the ELE area that explicitly aim at addressing these challenges.

The book is intended for use by different professionals from medical doctors, ICT specialists, mathematicians, engineers and programmers to caregivers and 
third parties like insurance companies' personnel and end users. It could be used as a notebook in the related curriculum. The chapters could be read in any order.

The overall objectives of this book are to:

- Offer an up-to-date analysis of architectures, techniques, protocols, components and development related to the AAL and ELE areas.

- Explain state-of-the-art technological solutions for the main AAL/ELE issues, stressing on computing and sensors.

- Demonstrate the importance of the emotional status of people in their living environment.

- Present the AAL/ELE benefits along with the development process of scientific and commercial applications and platforms.

The book's mission is to make readers familiar with these concepts and technologies that are successfully used in the implementation of today's AAL/ELE systems or are promising in future developments. The approach is more practical than theoretical, defining different concepts under a hierarchical reference model for ELE. The interdisciplinary nature of the topic brings together also an interdisciplinary team of authors that presented different views on the topic. The book contains many examples showing specific applications and highlighting possibilities for the integration on a generic platform.

The book presents also up-to-date technological solutions to the main aspects regarding AAL/ELE systems and applications including computing and artificial intelligence. The aim is to demonstrate the process started with AAL labs by further transforming these into islands with rich capabilities. This will allow crystallization and refinement of specifications and definitions, and will provide possibilities to offer appropriate services to broad public aiming at the same time to decrease the cost of their provision. The book discusses contemporary AAL/ELE technologies designed to solve some of the thorniest business problems affecting applications in areas such as health and medical supply, smart cities and smart houses, big data, Internet of Things (IoT) and many more.

The book consists of 14 chapters that are grouped logically depending on its respective topics. Chapter 1 is an introduction to ELE. Chapter 2 deals with ELE from the viewpoint of the socioecological psychology. It highlights an interesting approach towards the emotional state of people under monitoring. Chapter 3 explains pervasive sensing for social connectedness and enriches the topic of Chapter 2. Chapter 4 presents a specific view towards elderly, raising also some ethical issues.

Chapter 5 defines in a more technical way how the end users, AAL and ELE address service scenarios within a smart PLE. Chapter 6 demonstrates an interesting measurement of stress. An application of artificial intelligence in searching for health information is shown in Chapter 7.

Chapters 8 and 9 relate to communications and networking aspects of AAL/ELE platforms and systems along with their architectures and possible implementations. 
A broad analysis of the linear wireless sensor networks (LWSNs) and protocols for use in the next-generation networks is included in Chapter 10. A theoretical approach towards the actual software implementation on the target ELE platform is shown in Chapter 11. Chapter 12 presents the use of wireless body area networks for health monitoring along with corresponding challenges, topology design and thermal issues. Chapter 13 presents an interesting survey on the wearable healthcare technology. Chapter 14 presents a sophisticated intelligent system for afterstroke home rehabilitation. 
This page intentionally left blank 


\section{Acknowledgements}

This book would not have been possible without the help of many people. We would like to thank the reviewers, colleagues from IET, authors, co-authors and friends for their direct and indirect support.

Many special thanks to all colleagues from the EU COST action IC1303 'Algorithms, Architectures and Platforms for Enhanced Living Environments' (AAPELE) for their encouragement, advices, proposals and reviews. Many of them are authors and had been cited in the book.

Special thanks to Valerie Molière, Olivia Wilkins, Jennifer Grace and Paul Deards of IET Publishers as well as Srinivasan Natarajan of MPS Ltd. for their patience, corrections, advices and valuable support.

Book Editors 